\title{
Laparoscopic management of heterotopic pregnancy in an IVF conception
}

\author{
Nitin H. Shah, Riddhi J. Shah*, Swapnali Kshirsagar
}

Department of Obstetrics and Gynecology, Vardann Multispeciality Hospital, Mumbai, Maharashtra, India

Received: 01 January 2018

Accepted: 31 January 2018

\section{*Correspondence:}

Dr. Riddhi J. Shah,

E-mail: rydhie@gmail.com

Copyright: (C) the author(s), publisher and licensee Medip Academy. This is an open-access article distributed under the terms of the Creative Commons Attribution Non-Commercial License, which permits unrestricted non-commercial use, distribution, and reproduction in any medium, provided the original work is properly cited.

\begin{abstract}
Heterotopic Pregnancy cases are on the rise in the era of Artificial Reproductive Techniques and managing these pregnancies can be challenging especially in safeguarding the precious intrauterine pregnancy. These were traditionally managed by laparotomy and there are few cases reported wherein salpingectomy is done laparoscopically. We would like to report this case of a 7 weeks intrauterine pregnancy with 5 weeks tubal ectopic treated by laparoscopic salpingectomy under spinal anaesthesia while safeguarding the intrauterine viable gestation. In the light of increased incidence of abnormal implantations and growing demand and expertise of minimally invasive surgeries, laparoscopy is the treatment of choice for heterotopic pregnancies, especially in the interest of the intrauterine pregnancy.
\end{abstract}

Keywords: Heterotopic, Laparoscopy, Salpingectomy

\section{INTRODUCTION}

Heterotopic pregnancy is a dangerous condition with coexistence of an intrauterine gestation and an ectopic implantation elsewhere. The incidence of this condition is 1 in 30000 in the general population. ${ }^{1}$ It is a rare condition but increasingly reported after the advent of artificial reproduction in which the incidence is up to 1 in 125 , however it can also occur in few cases in a spontaneous pregnancy or after intrauterine insemination. $^{2}$

The risk factors in spontaneous pregnancy are pelvic inflammatory disease, genital Tuberculosis, previous ectopic pregnancy or a previous tubal surgery. It is a lifethreatening condition requiring surgical management which was traditionally by laparotomy but now increasingly made possible laparoscopically for a favourable intrauterine outcome as well as reduced patient morbidity. The following case highlights the principles of endoscopic surgery used for a delayed IVF conception of 7 weeks gestation within 5 weeks tubal unruptured ectopic.

\section{CASE REPORT}

Mrs A, 30-year-old with history of primary infertility underwent a second in vitro fertilisation by frozen embryo transfer on day 16 of menstrual cycle after a course of one cycle of combined hormonal contraceptive pills.

In view of previous one failed embryo transfer, four frozen embryos were transferred following which she was started on Folic acid, Tablet Estradiol valerate, low dose Aspirin, oral and vaginal micronized progesterone and tablet Sildenafil citrate. Beta-HCG levels were monitored and on day 20 of the transfer they were 147.3 $\mathrm{mIU} / \mathrm{ml}$ which increased to $350 \mathrm{mIU} / \mathrm{ml}$ after two days indicating a live gestation. Ultrasound on day 35 of the 
embryo transfer showed a $5.2 \mathrm{~mm}$ gestational sac in the uterine cavity corresponding to less than 5 weeks gestation which was suggestive of a delayed gestation.

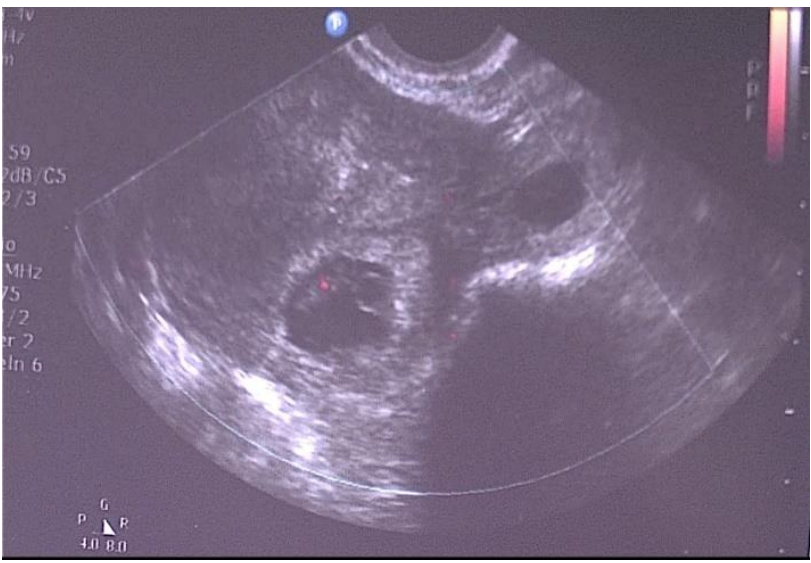

Figure 1: Ultrasound picture.

Follow up Ultrasound done two weeks later showed a live intrauterine pregnancy of 7 weeks 2 days with an extrauterine left adnexal mass lesion with an anechoic gestational sac measuring $1 \mathrm{~cm}$ corresponding to 5 weeks with minimal perilesional vascularity on color Doppler. Heterotopic pregnancy was the diagnosis made and laparoscopic salpingectomy was planned after counselling the couple.

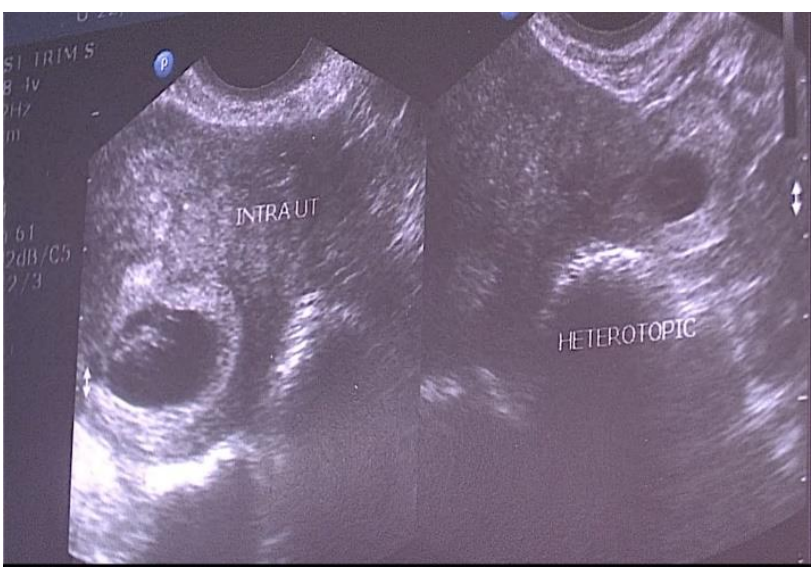

Figure 2: Intrauterine and tubal pregnancy on ultrasound image.

Patient had no past illnesses or comorbidity and all her basic investigations and examination was found to be normal. Aspirin was stopped a week in advance of the procedure. Patient was taken for laparoscopic total salpingectomy on the affected side under Spinal Anaesthesia after an informed consent by the couple and adequate preoperative measures.

Spinal Anaesthesia with intravenous sedation was administered after confirming level of anaesthesia. Primary $10 \mathrm{~mm}$ trocar was inserted through a supraumbilical incision by direct entry method and two
$6 \mathrm{~mm}$ accessory trocars were inserted under vision- left lower at a site $2 \mathrm{~cm}$ superomedial to the anterior superior iliac spine and left upper $10 \mathrm{~cm}$ above the left lower in the midclavicular line. Operation was performed at a gas pressure of $10 \mathrm{mmHg}$.

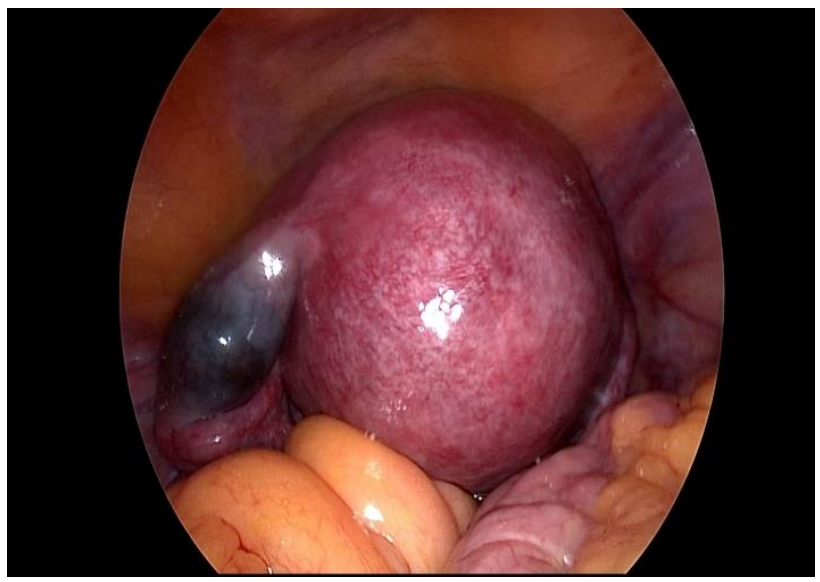

Figure 3: Intraoperative- Left isthmic ectopic with gravid uterus.

In-situ findings revealed a $4 * 2 \mathrm{~cm}$ unruptured left isthmic ectopic pregnancy with a bulky congested gravid uterus. There was no hemoperitoneum. Right adnexa and left ovary were found to be normal.

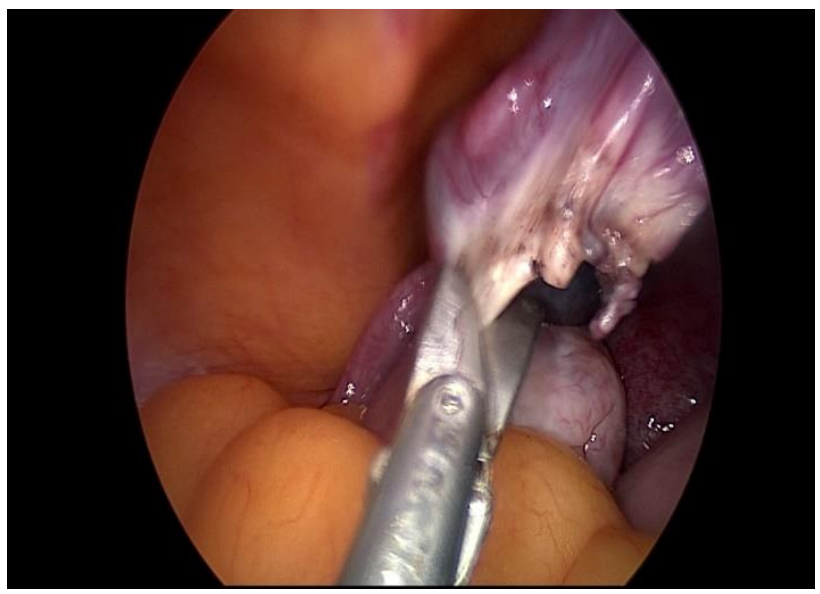

Figure 4: Salpingectomy with bipolar cautery and scissors.

Left fallopian tube was carefully lifted up with an atraumatic grasper and the mesosapinx coagulated with bipolar cautery throughout its length with careful cauterisation near the cornu as it was an isthmic ectopic. The base of the fallopian tube along with the ectopic pregnancy was cut with scissors and specimen retrieved by enlarging the left lower port through a $10 \mathrm{~mm}$ trocar. Hemostasis was confirmed, and normal saline wash given.

Postoperative patient was continued on folic acid, estradiol, micronised progesterone and aspirin started 
after 24 hours. Patient's condition was good, and Ultrasound done revealed live intrauterine gestation and otherwise normal pelvic sonography. Patient was discharged on day 3 and followed up a week later and thereafter after 2 weeks and is currently having 16 weeks normal pregnancy. Dual Marker test and Nuchal scan done at 12 weeks gestation was normal.

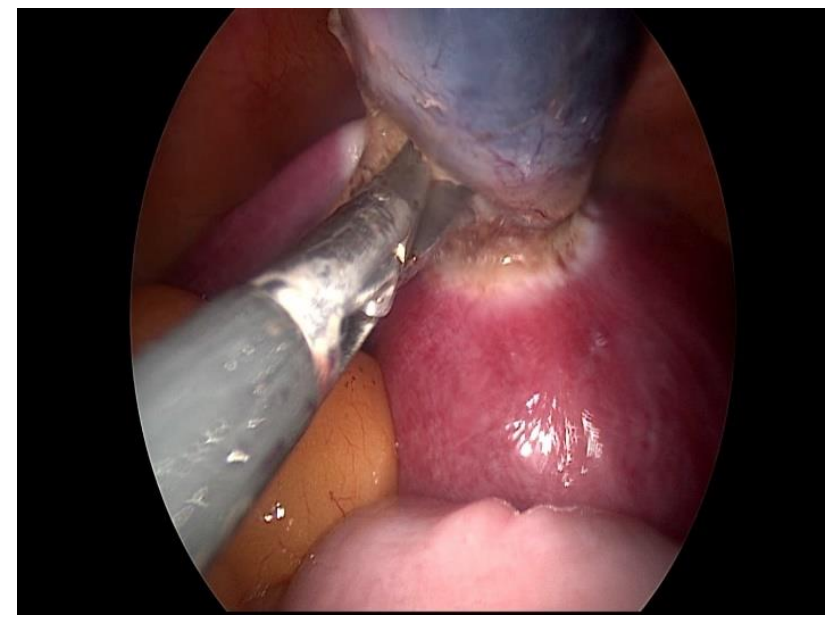

Figure 5: Careful coagulation and cutting near cornu.

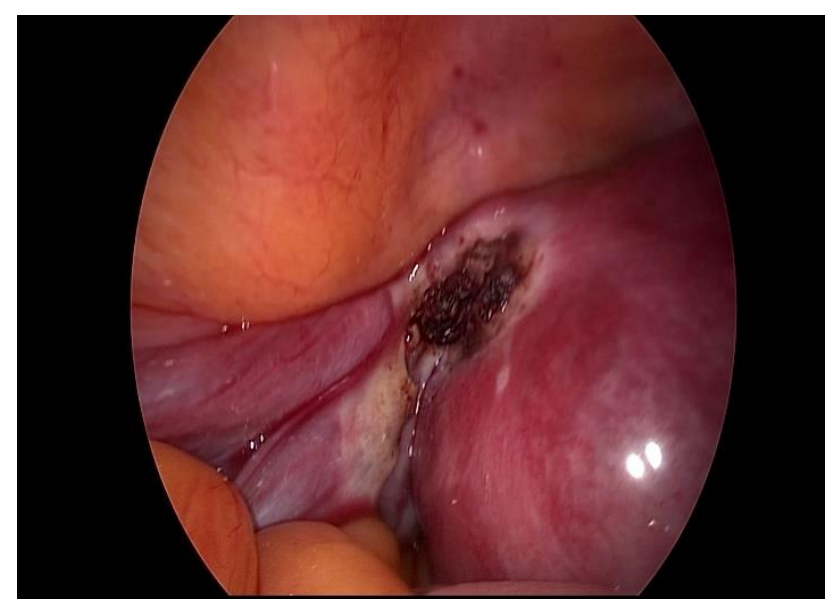

Figure 6: Postoperative image.

\section{DISCUSSION}

The simultaneous implantation of embryo intrauterine as well as at an ectopic site is termed as heterotopic pregnancy and its incidence with Artificial Reproductive Techniques has been estimated by some to be about $1 \%$ compared to 1:500000-1:10000 in natural conceptions. Mostly diagnosed by Ultrasound, $70 \%$ between 5 to 8 weeks of gestation. ${ }^{3}$

The presence of ectopic pregnancy is like a time bomb in the fallopian tube which can be life-threatening. It can cause loss of the desired pregnancy as well. Possible complications include tubal rupture, haemorrhagic shock, and the risk of spontaneous abortion, disseminated intravascular coagulation and death. Hence, it is important that it be diagnosed and treated early. BetaHCG doubling rate is 48 hours usually in a normal pregnancy however it may be normal doubling time in $17 \%$ heterotopic pregnancies and $15 \%$ intrauterine pregnancies may not show a normal doubling time. So, it cannot be relied on. Progesterone levels also does not distinguish ectopic pregnancies from failed intrauterine gestations. Ultrasound is the most common method of diagnosing. The differential diagnosis includes haemorrhage in corpus luteum or an ovarian cyst, especially in overstimulated ovaries in ART. A study showed definitive diagnoses being made at laparotomy or laparoscopy in $73 \%$ cases showing that it is a definitive method of diagnosing, although involves risk of surgery. ${ }^{4}$

Modality of treatment is elective surgery in most cases as it confirms the diagnosis, proves to be least harmful to the intrauterine foetus and avoid risk of rupture and haemorrhage. Other modalities include ultrasound guided injection of potassium chloride or $50 \%$ glucose, direct aspiration of products or an expectant management. ${ }^{5}$

Analysis of studies shows laparoscopic salpingectomy as a more suitable treatment than conservative laparoscopic surgeries like salpingostomy due to lesser risk of postoperative haemorrhage, persistent ectopic or recurrent ectopic. ${ }^{6}$ Laparoscopic technique is only slightly modified by the presence of the intrauterine gestation as minimal uterine manipulation must be ensured. Regional anaesthesia is preferred. Open technique of entry should be preferred. Operating insufflation pressure is kept as low as possible, preferably $<12 \mathrm{mmHg}$ and excessive Trendelenburg position avoided. End tidal $\mathrm{CO} 2$ should be monitored during the procedure along with other vital parameters. $^{7}$

A few similar cases have been reported in literature. A recent report from Kenya in 2016 used laparoscopic management under General Anaesthesia, used a sponge on forceps in the vagina, Verees high pressure insertion done at $18 \mathrm{mmHg}$ and operating pressure used was $15 \mathrm{mmHg} .{ }^{8}$ Cases have been reported of laparoscopic management in cases of acute abdomen and patient in shock suggestive of a ruptured tubal pregnancy. ${ }^{9}$ Although heterotopic pregnancy in second trimester is $<10 \%$ of the cases, there are cases reported managed by laparoscopy at that gestation too. ${ }^{10}$ Adequate surgical expertise and careful trocar placement and insertion can lead to good surgical outcomes in such cases. Considerable expertise and availability of a team of doctors in institution has made it possible to use endoscopic surgery for ruptured heterotopic pregnancy as well. ${ }^{1}$ In fact laparoscopy can also be used in the trained hand for rare cases of ruptured heterotopic cornual ectopic, as proved by a study in 2016 involving 13 cases of cornual resection done for heterotopic pregnancy. ${ }^{11}$

Review of these cases is done to measure foetal outcome as opposed to laparotomy or conservative management. Studies have shown a rise in the foetal survival rate over 
the years for heterotopic pregnancies managed laparoscopically. 50-60\% foetal survival was the standard in studies conducted in the 90s. ${ }^{6}$ Whereas a study in 2013 of 17 heterotopic pregnancies including 3 cornual heterotopic managed laparoscopically showed favourable neonatal outcome in all but 2. This suggests improved means of managing abnormal pregnancies that has led to less patient morbidity with an improved neonatal survival. ${ }^{12}$

\section{CONCLUSION}

In conclusion, we would like to stress that diagnosis and management of heterotopic pregnancy is challenging even with improved technology and knowledge in our era. With increasing incidence of the same, laparoscopy remains the most appropriate means of diagnosing and operating abnormal implantations in pregnancy including heterotopic pregnancy and that considerable expertise is essential for managing these cases.

Funding: No funding sources Conflict of interest: None declared

Ethical approval: Not required

\section{REFERENCES}

1. Morgan Gruner CR, Peter F XJ, Jiang X, Schnatz PF. Spontaneous heterotopic pregnancy: a case report. Gynecol Obstet. 2015 Sep;5(9).

2. Hutchinson M, Chan C. Laparoscopic management of ruptured heterotopic pregnancy after intrauterine insemination. CMAJ. 2016;188(17-18):E525-7.

3. Brown J, Wittich A. Spontaneous heterotopic pregnancy successfully treated via laproscopic surgery with subsequent viable intrauterine pregnancy: a case report. Military Med. 2012;177(10):1227-30.

4. Barrenetxea G, Barinaga-Rementeria L, Lopez de Larruzea A, Agirregoikoa JA, Mandiola M, Carbonero K. Heterotopic pregnancy: two cases and a comparative review. Fertil Steril. 2007 Feb;87(2):417.

5. Baxi A, Kaushal M, Karmalkar H, Sahu P, Kadhi P, Daval B. Successful expectant management of tubal heterotopic pregnancy Case Report. J Hum Reprod Sci. 2010 May;3(2):108.

6. Louis-Sylvestre C, Morice P, Chapron C, Dubuisson JB. The role of laparoscopy in the diagnosis and management of heterotopic pregnancies pregnancy (EP), and four had undergone previous tubal surgery. Hum Reprod. 1997;12(5):1100-2.

7. Reitman E, Flood P. Anaesthetic considerations for non-obstetric surgery during pregnancy. $\mathrm{Br} \quad \mathrm{J}$ Anaesth [Internet]. 2011 Dec;107(suppl_1):i72-8.

8. Mutiso, Kyende S, Mukaindo, Mwaniki A. Heterotopic pregnancy and subsequent pregnancy outcome: A case report. Int J Case Reports Images. 2016 Jun;7(8):508-13.

9. Goettler S, Zanetti-Dällenbach R. Heterotopic Pregnancy. N Engl J Med. 2016 Nov;375(20):1982.

10. Khan MN, Rafique S, Ranaboldo CJ. Laparoscopic management of heterotopic pregnancy: a rare cause of acute abdomen in women. Surg Laparosc Endosc Percutan Tech. 2009 Feb;19(1):e29-31.

11. Kim MJ, Jung YW, Cha JH, Seok HH, Han JE, Seong SJ, et al. Successful management of heterotopic cornual pregnancy with laparoscopic cornual resection. 2016 Aug 1;203:199-203.

12. Eom JM, Choi JS, Ko JH, Lee JH, Park SH, Hong $\mathrm{JH}$, et al. Surgical and obstetric outcomes of laparoscopic management for women with heterotopic pregnancy. J Obstet Gynaecol Res. 2013 Dec;39(12):1580-6.

Cite this article as: Shah NH, Shah RJ, Kshirsagar

S. Laparoscopic management of heterotopic pregnancy in an IVF conception. Int J Reprod Contracept Obstet Gynecol 2018;7:1255-8. 